\title{
THE RESEARCH ON ACCURACY EVALUATION METHOD OF THE TOMOGRAPHIC SAR THREE-DIMENSIONAL RECONSTRUCTION OF URBAN BUILDING BASED ON TERRESTRIAL LIDAR POINT CLOUD
}

\author{
Li Linze $^{\mathrm{a}}$, Pang Lei ${ }^{\mathrm{b}}$, Zhang XueDong ${ }^{\mathrm{b}}$, Liu Hui ${ }^{\mathrm{c}}$ \\ ${ }^{a}$ School of Geomatics and Urban Spatial Information, Beijing University of Civil Engineering and Architecture, yongyuan \\ street,Beijing-lilinze0115@163.com \\ ${ }^{\mathrm{b}}$ School of Geomatics and Urban Spatial Information, Beijing University of Civil Engineering and Architecture, yongyuan \\ street, Beijing-panglei@bucea.edu.cn \\ ${ }^{c}$ Telecommunications Institute, Beijing University of Civil Engineering and Architecture, yongyuan \\ street, Beijing
}

KEY WORDS : Tomographic SAR, Lidar, Urban Building, 3d Point Cloud, Facade Reconstruction ,Accuracy Evaluation.

\begin{abstract}
:
Muti-baseLine SAR tomography can be used on 3D reconstruction of urban building based on SAR images acquired. In the near future, it is expected to become an important technical tool for urban multi-dimensional precision monitoring. For the moment, There is no effective method to verify the accuracy of tomographic SAR 3D point cloud of urban buildings. In this paper, a new method based on terrestrial Lidar 3D point cloud data to verify the accuracy of the tomographic SAR 3D point cloud data is proposed, 3D point cloud of two can be segmented into different facadeds. Then facet boundary extraction is carried out one by one, to evaluate the accuracy of tomographic SAR 3D point cloud of urban buildings. The experience select data of Pangu Plaza to analyze and compare, the result of experience show that the proposed method that evaluating the accuracy of tomographic SAR 3D point clou of urban building based on lidar 3D point cloud is validity and applicability
\end{abstract}

At the end of twentieth Century, tomography technology is introduced in synthetic aperture radar, and produce the Tomographic SAR. Compared with the traditional SAR imaging technology, Tomography SAR imaging technology as a three-dimensional high-resolution SAR imaging system in addition to the range and azimuth to have a higher resolution, while increasing the ability to distinguish in elevation. It really achieved the three-dimensional imaging of the target, in recent years it's more and more attractive for many domestic and foreign research institutions and researchers. Three-dimensional reconstruction by the SAR will inevitably produce some errors, the error mainly includes time coherence error, SNR error, polarization error, atmospheric phase difference, etc. The influence of these factors is reflected in the accuracy of the point cloud data. The accuracy of the analytical SAR point cloud assessment mainly refers to the evaluation of plane accuracy and elevation accuracy, also known as the positioning accuracy evaluation. The accuracy of the SAR data is critical to the subsequent data processing and quality of the results. The terrestrial lidar is a set of global navigation system, inertial navigation system and lidar scanner in one for three-dimensional information remote sensing system. lidar as a new means of information access, it has a high point cloud density, fast efficiency, little impact by the weather, high data accuracy, rich product advantages, etc ${ }^{[1]}$. So the using of the current relatively mature LIDAR technology can be very good to verify the accuracy of the tomographic SAR.

\section{TOMOGRAPHICSAR THREE-DIMENSIONAL RECONSTRUCON}

Compared with the traditional SAR, the tomographic SAR adds a synthetic aperture at elevation, it achieve high-resolution scatterer to high-resolution imaging by multi-baseline flighting over the same area. Three-dimensional imaging of the observed area is currently done mainly through the existing multi-orbit flight of the SAR system. First of all, it is necessary to observe the same area at different heights, to complete the focusing on range and azimuth for surface features. And the most critical focus on elevation need to observe the different tracks on the data and collected to calculate. Assume that Single Look Complex Images obtained from a different height orbit is observed for a region, and then to these images for image registration, de-slope and other pretreatment. After the steps we can choose a scatter as an example, the imaging results can be expressed by the following formula:

$$
\begin{aligned}
& \mathrm{g}(\mathrm{n})= \gamma(\mathrm{S}) \exp (\mathrm{j} 2 \quad) d \\
&-\quad, \quad \mathrm{n}=1,2, \ldots \ldots \ldots \ldots \ldots, \mathrm{N}
\end{aligned}
$$

$\gamma(\mathrm{S})$ is the distribution function of the complex scatter coefficient along the nsr direction, $\lambda$ is wavelength, $R$ is the slant-range of the image center, is a vertical baseline. When the vertical baseline is evenly sampled, is sampling interval, - According to Nyquist Theorem, When the sampling interval is $\Delta \mathrm{f}$, The domain of no ambiguity time that can be reconstructed is $2 \mathrm{t} \leq 1 / \Delta \mathrm{f}$. Apply to the above process, the ambiguity time domain with nsr direction is 2 smax. 2 smax $=$ -. [-smax, smax] is span of nsr, the length of equivalent synthetic aperture at nrs is determined by the vertical baseline span $\mathrm{B}$. The nsr to Rayleigh resolution corresponding to the equivalent synthetic aperture is $\rho_{\mathrm{s}}=2 \mathrm{~B} / \lambda \mathrm{r}$.

When the three dimensions of the building are reconstructed by tomographic SAR, Due to the limitation of SAR satellite orbit and the lack of satellite image, the location error of TomoSAR points is highly anisotropic with an elevation error typically one

\footnotetext{
b Corresponding author Pang Lei ,email address, panglei@bucea.edu.cn
} 
or two orders of magnitude higher than in range and azimuth ${ }^{[2]}$. In addition, because there will be some complex pseudo-scatterer around the building, SAR satellites will also receive electromagnetic signals from these scatterers when they fly over the building, some abnormal points away from the actual location of the building will be generated. The two-dimensional images of the SAR is coherent, so compared with the lidar when the three-dimensional reconstruction of the building through the TomoSAR more emphasis on the characteristics of the building. Side-looking SAR geometry enables TomoSAR point clouds to possess rich facade information. At the same time, objects such as trees, which are not coherent, will not be reconstructed ${ }^{[4-6]}$. This avoids the influence of the surrounding environment on the three-dimensional reconstruction of buildings. In addition, the three-dimensional point cloud of the building through TomoSAR can obtain the Scattering characteristics of scatterers from these characteristics, we can get the information of the material and geometry of the building facade.

\section{FUNDAMENTALS OF TERRESTRIAL LIDAR}

Just as its name implies, the ground 3D laser radar scanning system is on the ground, similar to the traditional ground photogrammetry ${ }^{[3]}$. It uses non-contact high-speed laser measurement, the geometric shape data of $3 \mathrm{D}$ terrain and complex objects are obtained in the form of point cloud. Laser scanner mainly includes laser ranging system and laser scanning system, At the same time, it also integrates CCD and instrument internal control and calibration system. In the instrument, the two laser beams can be rotated in a fast and orderly manner, and the narrow laser pulse emitted by the laser pulse emitter is swept over the measured area, The distance of each laser pulse is calculated from the time difference (or phase difference) that is emitted from the surface of the analyte and then returned to the instrument, At the same time, the scanning control module controls and measures the angle of each pulse laser, and finally calculates the three-dimensional coordinates of the laser spot on the measured object.

\section{ACCURACY EVALUATION OF TOMOSAR BUILDING RECONSTRUCTION BASED ON THE TERRESTRIAL LIDAR}

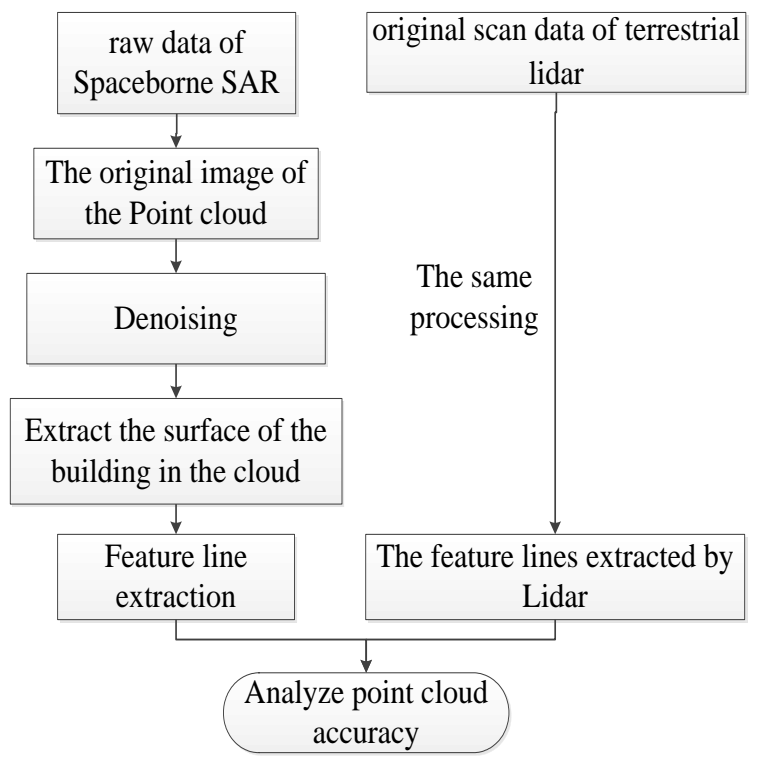

Fig3-1 Flow Chart of Accuracy Evaluation
Point location of point cloud data is not easy to directly use measured instrument to obtain, the appropriate method should be chosen to evaluate the accuracy of point cloud. Commonly used methods are point and point comparative analysis, statistical analysis and so on. Since the lidar point cloud and the TomoSAR point cloud are all described as the location of the irregular distribution of the target surface, Point cloud density is related to many conditions. Therefore, it is difficult to find the points corresponding to the feature points accurately in both points. The accuracy of the terrestrial Lidar scan data can reach the millimeter level, it can be very accurate to build the outline of the building, So we can verify the accuracy of the point cloud by comparing and analyzing the geometric characteristics of the point cloud. For millimeter level ground lidar scan data, there is already a very mature processing flow. This paper mainly discusses the processing of the TomoSAR point cloud. First of all, we need to filter the TomoSAR point cloud, and the original TomoSAR point cloud will have a lot of messy points around the target point cloud, these will affect the subsequent extraction of the building contour. Through the filter, you can remove some outliers in the point cloud, Its principle is to filter the original point cloud of each point to traverse, delete all the data points within a certain range without reaching enough neighbors, this distance is a threshold that needs to be set by itself. After this step, we can get a complete point cloud model without isolated point. After obtaining the point cloud model of the building after filtering, as the point cloud data volume is relatively large, the overall participation in the operation makes the occupation of resources is relatively large, it is necessary to perform the grid processing of the TomoSAR cloud model. The role of the establishment of the grid in addition to facilitate the processing of data, by analyzing the relationship between adjacent grid, for the follow-up point cloud model processing also has a great matter. When building a virtual grid element for point cloud data, first count the original point cloud data set point cloud number and point cloud occupied by the horizontal area, the overall density of the estimated point cloud is points $/ \mathrm{m}^{2}$. And then according to the point cloud density roughly determine the grid spacing, for the points in the original TomoSAR data set, the index between the grid and the grid is established. Then traverse all the grid, search the index corresponding to the point cloud, statistical point cloud density and maximum elevation value. It is judged whether the point cloud density and the maximum elevation value of the grid satisfy the threshold value, and if it is satisfied, it is added to the building point cloud data set, else it is given out. Then complete the building point cloud recognition extraction. After this step, it is necessary to segment the point cloud of the building, and separate the different facades of the building. Based on the characteristics of the scattered distribution of cloud points in the TomoSAR, the adaptive RANSAC algorithm is used here, because the building elevation is mostly perpendicular to the ground, the normal vector is 
perpendicular to the vertical direction, so the normal vector constraint condition is increased. It can be more accurate segmentation of the TomoSAR building facade point cloud. Then use least squares algorithm for each segment of the facade of the point cloud plane fitting, after divided the facade of the building, and determine its plane parameters. The intersection is obtained by intersecting the surface with the surface, it can be directly obtained after the rule of the vertical boundary. The general equation of space plane is expressed as

$$
A x+B y+C z+D=0
$$

In the formula, A, B, C, D are parameters of plane. The number of points in each elevation due to segmentation is far greater than 3 , and the discrete distribution of point cloud on both sides of the plane, In order to obtain the intersection between planar patches, it is necessary to have a plane fitting for point cloud of facades. It can use least square algorithm. The best plane is obtained on the basis of the least square sum of errors. Below is Least square plane fitting algorithm:

In this paper, the vertical and the ground buildings are used to detect the accuracy of TomoSAR point cloud, its facades perpendicular to the ground, so all points in the plane equation can be expressed as follows:

$$
\left\{\begin{array}{c}
z_{1}=A x_{1}+B y_{1}+D \\
z_{1}=A x_{2}+B y_{2}+D \\
\vdots \\
\vdots \quad \vdots \quad \vdots \\
z_{1}=A x_{n}+B y_{n}+D
\end{array}\right.
$$

Suppose there are $\mathrm{n}$ points, $\left(x_{n}, y_{n}, z_{n}\right)$ is coordinate of each point, it can be expressed in the form of a matrix:

$$
\begin{aligned}
& {\left[\begin{array}{ccc}
x_{1} & y_{1} & 1 \\
x_{2} & y_{2} & 1 \\
\vdots & \vdots & \vdots \\
x_{n} & y_{n} & 1
\end{array}\right]\left[\begin{array}{l}
A \\
B \\
D
\end{array}\right]=\left[\begin{array}{c}
z_{1} \\
z_{2} \\
\vdots \\
z_{n}
\end{array}\right] \quad P X=Q} \\
& P=\left[\begin{array}{ccc}
x_{1} & y_{1} & 1 \\
x_{2} & y_{2} & 1 \\
\vdots & \vdots & \vdots \\
x_{n} & y_{n} & 1
\end{array}\right], \quad Q=\left[\begin{array}{c}
z_{1} \\
z_{2} \\
\vdots \\
z_{n}
\end{array}\right],
\end{aligned}
$$

$\mathrm{X}$ is plane parameter $(A, B, D)$, and $\mathrm{C}=1$.

The least squares solution of the plane parameters are as follows:

$$
X=\left(P^{T} P\right)^{-1} P^{T} Q
$$

Repeat this process for each facaded, the plane parameters of all facades can be obtained, then the intersection line is obtained according to the plane equation of the adjacent two facades. The two facade equations are as follows:

$$
\left\{\begin{array}{l}
A_{1} x+B_{1} y+C_{1} z+D_{1}=0 \\
A_{2} x+B_{2} y+C_{2} z+D_{2}=0
\end{array}\right.
$$

The normal vector is

$$
\vec{a}=\left(A_{1}, B_{1}, C_{1}\right), \vec{b}=\left(A_{2}, B_{2}, C_{2}\right)
$$

Intersect the line equation is

$$
\frac{x-M}{E}=\frac{y-N}{F}=\frac{z}{G}
$$

The direction of the intersecting line can be obtained by crossing the plane normal vector

$$
\begin{aligned}
\vec{a} \times \vec{b}=\left|\begin{array}{ccc}
i & j & k \\
A_{1} & B_{1} & C_{1} \\
A_{2} & B_{2} & C_{2}
\end{array}\right| & =\left(B_{1} C_{2}-B_{2} C_{1}\right) i+\left(A_{2} C_{1}-A_{1} C_{2}\right) j+\left(A_{1} B_{2}-A_{2} B_{1}\right) k \\
& =(E, F, G)
\end{aligned}
$$

The point can be obtained through the intersecting line intersect the XOY plane, it's $(M, N, 0)$ Then the simultaneous equations can be obtained:

$$
\left\{\begin{array}{l}
M=\frac{B_{1} D_{2}-B_{2} D_{1}}{A_{1} B_{2}-A_{2} B_{1}} \\
N=\frac{A_{1} D_{2}-A_{2} D_{1}}{A_{2} B_{1}-A_{1} B_{2}}
\end{array}\right.
$$

So that the equations of the contours of the intersecting buildings can be extracted. After the equationis of plane obtained, it is easy to find the intersection between the faces, and then the extremes of the coordinates of each point in the direction of the point cloud can be used to obtain the coordinates of the characteristic line, and the coordinates of the endpoint can be obtained The length of the characteristic line.

\section{EXPERIMENT AND RESULT ANALYSIS}

In order to analyze the precision verification method proposed in this paper, the results of the measured data are given below. The TomoSAR data were selected from the terraSAR data from 2011 to 2014, it has a total of 14 tracks of data. Target is located in Beijing, Chaoyang District, China Pangu Seven Stars Hotel, The buildings selected for the experiment were flat rectangular buildings with a surface around them, its surface material for the reinforced concrete material, it has good reflection characteristics, and can be well built by the point cloud. Lidar data selected FARO laser radar scanner to obtain the point cloud data. A total of four stations were scanned by a laser radar scanner for the observation building, and then register the four stations together to form a complete point cloud. Use the methods used in this article to extract the contours of the building. Figure 1 shows the point cloud obtained from lidar, figure 2 shows the point cloud data of building obtained from the TomoSAR. Figure 3 is a comparison of lidar and TomoSAR acquisition of building data, from the figure we can see that Lidar points are more ordered points, and the point of the cloud generated by the sampling of the cloud point of chaos disorderly. And the point of the TomoSAR is relatively chaotic disorder. 


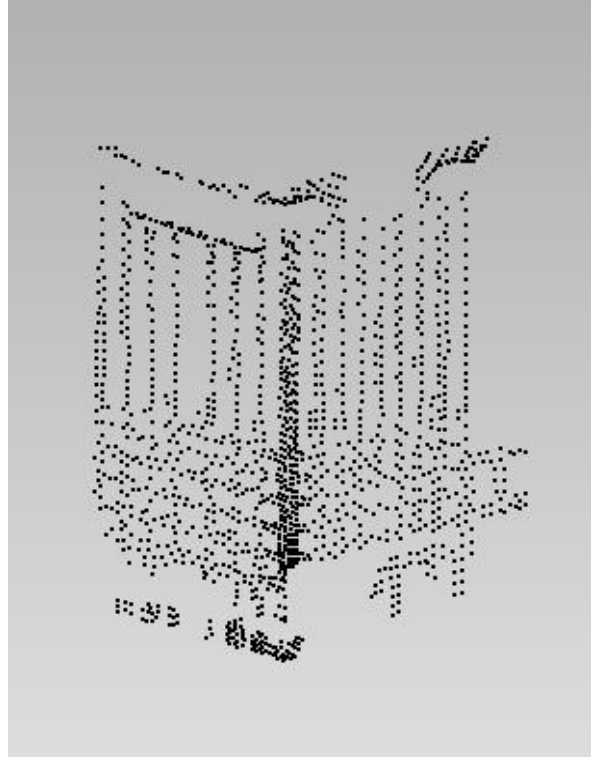

Fig4-1.Point Cloud Obtained From Terrestrial Lidar

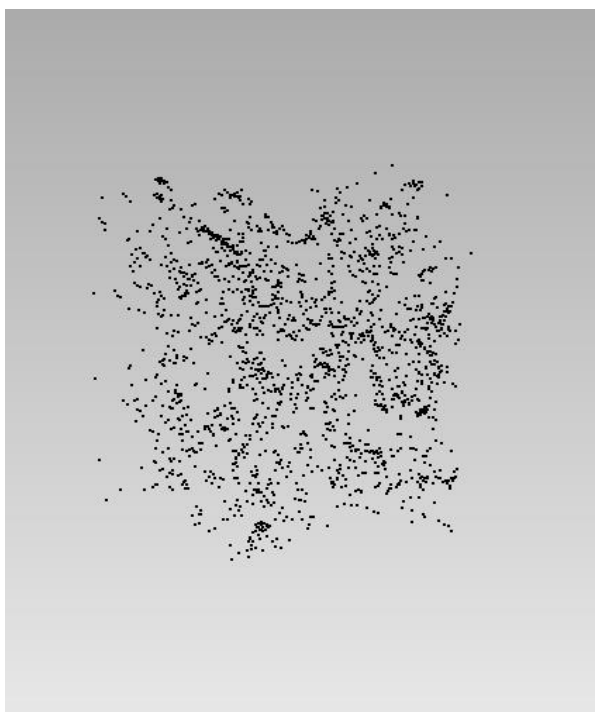

Fig4-2.Point Cloud Data Of Building Obtained From The Tomosar

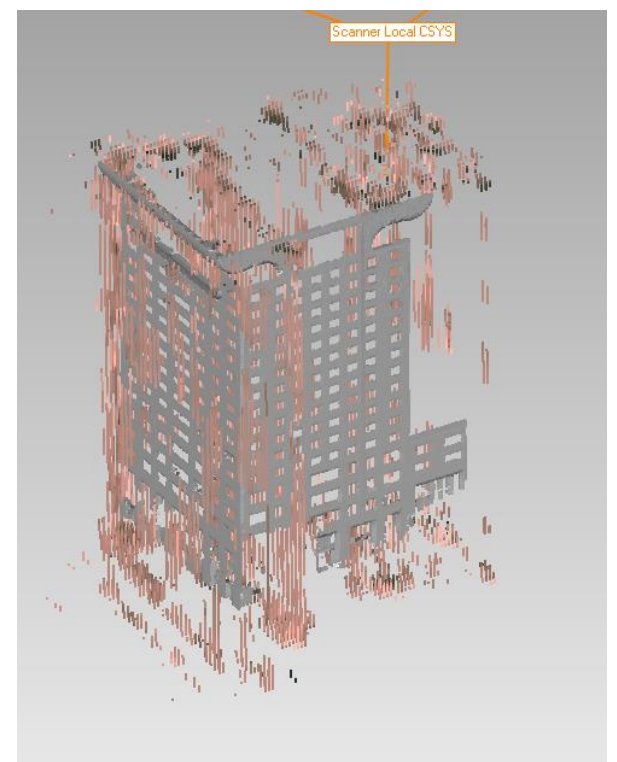

Fig4-3.. Comparison Of Lidar And TomoSAR Acquisition Of Building Data

The experiment can extract the two facades of the building extracted from the lidar and the ground of equation

$$
\begin{aligned}
& -0.0038 x+0.027 y+0.00035 z+1=0 \\
& 0.005 x+0.006 y+4.022 * 10^{-5} * z+1=0 \\
& 0.0022 x+0.004 y-0.0042 z+1=0
\end{aligned}
$$

At the same time, the three-faceted equations reconstructed from the reconstructed building point cloud model of the TomoSAR can be extracted as $-0.09 \mathrm{x}+9.02 * 10-17 * \mathrm{y}-5.55 * 10^{-17} * \mathrm{z}+1=0$
$0.0026 \mathrm{x}-0.01 \mathrm{y}-0.001 * \mathrm{z}+1=0$

The equations of the three surfaces can be obtained, and the straight line equation of the building facade and the two contours of the building can be obtained. Then the maximum and minimum values of $\mathrm{X}, \mathrm{Y}$, and $\mathrm{Z}$ are taken to obtain the height, length and width of the building. The results are shown in the following table

\begin{tabular}{|l|l|l|}
\hline \multicolumn{1}{|c|}{$(\mathrm{m})$} & TomoSAR & $\begin{array}{l}\text { Terresrial } \\
\text { lidar }\end{array}$ \\
\hline length & 47.03 & 47.05 \\
\hline width & 41.25 & 41.22 \\
\hline height & 90.10 & 88.184 \\
\hline
\end{tabular}

Table 1. The Results Of Experiment

\section{CONCLUSION}

Based on the experimental results, It can be seen that the results of three-dimensional reconstruction of the TomoSAR are similar to the building point cloud of lidar, and there is no great error in length and width, It is shown that the errors of the TomoSAR in azimuth and range are within the acceptable range, it can complete the reconstruction of the building. And the error of evelation is relatively large, the experimental results are consistent with the predicted results, this shows that through the lidar can be a good verification of the accuracy of the 
TomoSAR point cloud. The experiment needs further consideration is due to conditions, this experiment only selected a building as an goal of experiment, the number of test data is small, only the accuracy of the point cloud of TomoSAR can be roughly checked. If conditions permit, should increase the number of target buildings, has reached a more complete validation effect. TomoSAR is a fast, efficient and new method of data acquisition. However, due to the limitation of precision, the TomoSAR imaging algorithm needs to be further improved in order to improve its accuracy.

\section{ACKNOWLEDGEMENTS}

I would like to express my gratitude to all those who have helped me during the writing of this thesis. I gratefully acknowledge the help of my supervisor Pang Lei. I do appreciate her patience, encouragement, and professional instructions during my thesis writing. Also, I would like to thank Mrs He Shuguang and Miss Ai Liping, who kindly gave me a hand when I was making the questionnaire among the college English learners. Last but not the least, my gratitude also extends to my family who have been assisting, supporting and caring for me all of my life.

\section{REFERENCES}

1.Y.Wang, A.Xiong,J.,2013a.The research on Accuracy Evaluation Method of the Airborne Lidar Point Cloud
Data.Geomatics and Spatial Infortation Technology, 36(8),pp.130-131.

2. X. Zhu, M. Shahzad and R. Bamler,J., "From TomoSAR Point Clouds to Objects: Facde Reconstruction", Proceeding of theTyWRRS 2012, Naples, Italy.

3 S.Zhu,J.,2006a,The Technique Principle of LIDAR and Its Application in Surveying and Mapping. Modern Surveying and Mapping, 29(4),pp.12-13.

4 Rottensteiner F., Briese Ch., "A new method for building extraction inurban areas from high resolution LIDAR data", ISPRS. Photogrammet.Comput. Vision, Graz, Austria, pp. A-295 ff, 9-13 September, 2002.

5 F. Lombardini, "Differential Tomography: A New Framework for SAR Interferometry", In proceedings of IGARSS, Toulouse, France, pp.1206-1208, 2003.

6.A Shahzad M, Zhu X X. Reconstruction of building façades using spaceborne multiview TomoSAR point clouds[C]. international geoscience and remote sensing symposium, 2013: 624-627. 